ISSN: 2174-5609

DOI. http://dx.doi.org/10.14461/INTURI2017.13.05

FTr Investigaciones

MURISTICAS

\title{
Límites en la capacidad de carga de visitantes de ecoturismo en Punta del Este, Isla de la Juventud (Cuba)
}

\author{
Douglas Crispin Castellanos \\ douglas@cuij.edu.cu \\ Facultad de Cultura Física de la Universidad Jesús Montane Oropesa de Isla de la Juventud. Cuba.
}

Vicente Berovidez Álvarez

bero@fbio.uh.cu

Facultad de Biología. Universidad de La Habana. Cuba.

Jorge Marín Clemente

marim@inder.cu

Universidad de Ciencias de la Cultura Física y Deportes Manuel Fajardo de La Habana. Cuba

Francisco Enrique García Ucha

ucha@inder.cu

Universidad de Ciencias de la Cultura Física y Deportes Manuel Fajardo de La Habana. Cuba.

Juan Carlos Fernández-Truan

jcfertru@upo.es

Facultad de Ciencias del Deporte de la Universidad Pablo de Olavide de Sevilla. España.

\section{RESUMEN}

Esta investigación está encaminada a estudiar las capacidades de carga o límites aceptables de cambio, en la cantidad de visitantes que puede soportar una actividad ecoturística en una zona determinada, para contribuir a la conservación del medioambiente mediante un desarrollo sostenible. En este caso, el estudio se realizó en la zona sur de la Isla de la Juventud (Cuba); concretamente en la denominada Punta del Este. Para ello se utilizó una metodología mixta basada en métodos descriptivos: analítico-sintético y lógico abstracto, así como mediante el análisis experimental basado en el método desarrollado por Cifuentes en 1992 y mejorado en 1999. La aportación de esta investigación ha sido la obtención de datos sobre el mayor impacto de visitantes, o capacidad de carga que puede soportar la zona en las caminatas ecoturísticas realizadas por el turismo nacional e internacional, a fin de poder analizar las posibilidades económicas que este tipo de actividades pueden aportar, conservando su carácter de economía sostenible y sin que se comprometa el medioambiente para que las futuras generaciones puedan disfrutar y admirar la belleza de los recursos naturales existentes.

Palabras clave: turismo sostenible; medioambiente; deporte; senderismo 


\title{
Limits of acceptable change of ecotourism in Punta del Este, Isla de la Juventud (Cuba)
}

\begin{abstract}
The aim of this research is to study the carrying capacity or limits of acceptable change with respect to the number of visitors that an ecotourism activity in a specific area can bear so as to contribute to environmental conservation through sustainable development. The area chosen for this study corresponds to the southern region of Isla de la Juventud (Cuba); particularly in the so-called Punta Este. A mixed methodology was used, combining different descriptive methods: Analytic-Synthetic and logical abstract methods, as well as the experimental analysis based on the method developed by Cifuentes in 1992 and improved in 1999. The principal contribution of this work is to provide data on the maximum visitor impact, or the carrying capacity that this area can bear as a result of the ecotourism walking tours undertaken by domestic and international tourists. This research aims to analyse the financial gains that these types of activities can provide, maintaining their sustainable economic nature without causing negative impacts to the environment so that future generations can enjoy and admire the beauty of the existing natural resources.
\end{abstract}

Keywords: sustainable tourism; environment; sport; hiking

\section{INTRODUCCIÓN. CAPACIDAD DE CARGA Y ECOTURISMO HACIA UN DESARROLLO SOSTENIBLE}

La creciente demanda de los turistas por visitar destinos peculiares y especialmente áreas protegidas en todo el mundo, genera una preocupación global en los propietarios y administradores de estos lugares por la urgente necesidad de generar fondos económicos y recursos humanos capacitados que le permitan mantener la integridad de sus áreas. Según datos publicados por la Organización Mundial del Turismo (OMT), "la región de las Américas registró el mayor crecimiento relativo de todas las regiones del mundo en 2014 (+8\%), recibiendo 13 millones más de turistas internacionales que elevaron a 181 millones el total de visitas..." (OMT, 2015, p.7).

La importancia del turismo recreativo en los últimos años ha ido incrementándose hasta convertirse, en muchos países, en un factor fundamental de la economía, entendiendo impacto económico como "la medida de los beneficios y, también, de los costes económicos generados por el desarrollo de esta actividad" (Picornell, 2093, p.70). Este incremento obliga a tomar medidas para controlar los impactos negativos del turismo en áreas sensibles. Entre los elementos más importantes para el control de visitantes en las áreas silvestres protegidas están: la zonificación, el adecuado establecimiento de los espacios de visita, su capacidad de carga turística y la identificación de los impactos y su mitigación.

El concepto de capacidad de carga surge en un contexto ecológico y establece la capacidad de un sistema de soportar una población de tamaño determinado en relación a su nicho ecológico (espacio, nutrientes, luz, alimentos, refugio, competencia, etc.).

Al respecto, en la década de los 70 del pasado siglo, el concepto se utilizó por diversos autores para expresar la carga ambiental (Maldonado, Hurtado y Saborío, 1992). No 
fue hasta los 80 que Dunkel lo aplica al turismo. Analizó la progresión en el número de turistas que llegaban a las Islas Vírgenes y Bahamas, estableciendo curvas sigmoideas (semilogarítmicas) como las que describen el crecimiento poblacional de una especie, alcanzando un número de individuos (K) que responde a la capacidad de carga del ecosistema (Dunkel, 1984). Concluye Dunkel, que aun cuando no es directamente comparable, con este procedimiento de control se lograba un antecedente importante para establecer límites máximos a los volúmenes de turistas que visitan un espacio natural y los límites que pueden llegar a afectar ese destino.

La relación de este concepto con la sustentabilidad la estableció Sadler (1988). Por otro lado, Holder (1988) valoró que el concepto involucraba dos ámbitos fundamentales: por un lado, aquel que afecta directamente a los recursos, tanto por impacto producido a los ecosistemas como al bienestar de los propios turistas $y$, por otro lado, la calidad de la experiencia turística. Posteriormente Clark (1990) agregó un tercer ámbito, el de la capacidad de carga social.

Cifuentes ofreció en 1992, un procedimiento metodológico que aglutinaba la experiencia de varios años en el tema, con trabajos en el Parque Nacional Galápagos en Ecuador y Reserva Biológica Caracara en Costa Rica, entre otros. Sus principales aportaciones fueron por un lado que el procedimiento a aplicar es comprensible, sencillo y útil para determinar la capacidad de carga del área protegida en estudio y por otro, una propuesta de tres niveles de capacidad de carga turística, uno de los cuales considera la capacidad de manejo del área de estudio. En 1999 el propio Cifuentes corrigió su metodología, siendo esa propuesta el procedimiento metodológico empleado en este estudio.

Otros investigadores identificaron debilidades en los estudios de capacidad de carga. Diseñaron otras herramientas para enfrentar el problema como definir niveles de impacto y establecer criterios de manejo flexible sin definir números exactos de turistas (Graefe et al., 1990), surgiendo con ello el Visitor Impact Management (VIM).

Por otra parte, Stankey et al. (1985) establecieron otra propuesta mundialmente reconocida como el método de definición de Limites de Cambio Aceptable (LAC), basado en la definición de estándares de calidad de un área natural, que identifica la gama de oportunidades existentes en áreas naturales centrándose en definir hasta donde los cambios son aceptables para cada clase de oportunidad, para impedir que estos niveles se sobrepasen. Otros estudios a considerar son el Visitors Action Management (VAM) y Recreation Oportunity Spectrum (ROS).

La aplicación de estos criterios ha contribuido a afrontar el tema con rigor, pero cada vez se hace más evidente que el concepto debe ampliarse hacia la evaluación de la intensidad de uso turístico ambiental, a través de un adecuado control de visitantes y la cuantificación de sus impactos (Soria-Díaz y Soria-Solano, 2015; Gil et al., 2014).

La utilización de cualquiera de los métodos de estudio de capacidad de carga citados anteriormente, que buscan mitigar el impacto negativo de los visitantes a zonas de uso público para el desarrollo de actividades de recreación en la naturaleza, es uno de los elementos más importantes para lograr el desarrollo económico sostenible de poblaciones rurales situadas dentro o cerca de esas zonas, contribuyendo a la conservación de esas áreas naturales protegidas. 
En este sentido, se ha de considerar el ecoturismo como "la ejecución de un viaje a áreas naturales que están relativamente sin perturbar o contaminar, con el objetivo específico de estudiar, admirar y disfrutar del panorama junto con sus plantas, animales silvestres y cualquier otra manifestación cultural (pasada o presente), que se encuentre en esas áreas..." (Ceballos-Lascurain, 1996, p.226).

Para que una excursión en la naturaleza sea considerada ecoturismo, debe realizarse con unos objetivos bien definidos como: contemplación, admiración, disfrute, estudio, respeto y conservación de los recursos que la naturaleza ofrece al ser humano, a fin de que las futuras generaciones puedan llevar a cabo los mismos objetivos en el mismo contexto medioambiental, para poder lograr el verdadero desarrollo sostenible al que aspira la sociedad actual.

Además, el ecoturismo no debe valorar solamente los recursos naturales de un área, sino que debe considerar en conjunto los valores naturales, históricos y culturales que encierra la zona.

\section{ECOTURISMO EN LA ISLA DE LA JUVENTUD}

En Cuba existen zonas donde se ha empezado a dar los primeros pasos sobre el uso del impacto de visitantes en la sostenibilidad de un espacio natural; como por ejemplo en el Valle de Viñales en Pinar del Río, la Ciénaga de Zapata en Matanzas, algunas zonas de la Sierra Maestra (Sierra Maestra, 2008) y algunos cayos en Ciego de Ávila y Villa Clara (Najarro Pujol, 2011), pero no se ha realizado dentro de un análisis pormenorizado que estudie la capacidad de carga, sino como estudios aislados de ciertas giras especializadas (Empresa Nacional de Flora y Fauna, 1992; Jané, 1995; Medina y Santamaría, 2004).

Figura 1. Localización en azul de Isla de la Juventud en Cuba

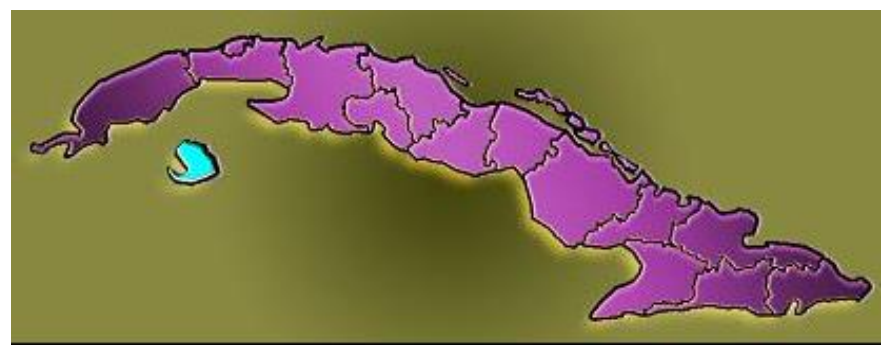

Elaboración propia

Para desarrollar la presente investigación se tomó como referencia las cinco regiones naturales en las que en 1967 se dividió la Isla de la Juventud (Cuba), escogiéndose para realizar este estudio la región meridional que se extiende al sur de la Ciénaga de Lanier (declarada sitio RAMSAS). Se trata de una zona llana muy poco accidentada que no sobrepasa los $10 \mathrm{~m}$ sobre el nivel del mar y que está compuesta por un suelo con una cubierta de rocas calizas de poca profundidad.

Toda esta región estuvo cubierta originariamente por un bosque denso, rico en maderas preciosas y con una gran diversidad vegetal. La zona litoral está formada por tres tipos diferentes de vegetación, con extensas playas e importantes cuevas, que históricamente demuestran la presencia de aborígenes en la zona durante épocas primitivas. 
Se trata de una extensa área en la naturaleza, que posee hermosos paisajes y otros valores de interés científico y cultural de gran importancia medioambiental, tanto a nivel nacional como internacional. En este espacio, habita una gran abundancia y diversidad de aves endémicas, por lo que también posee un gran valor para la observación de aves.

En esa porción sur de la Isla de la Juventud, también se encuentra otra zona con grandes potenciales para el ecoturismo, contando con gran biodiversidad de flora y fauna, excelentes playas de aguas cristalinas y magníficos fondos coralinos, sobresaliendo la playa de Punta Francés.

Figura 2. Localización de Punta del Este en Isla de la Juventud

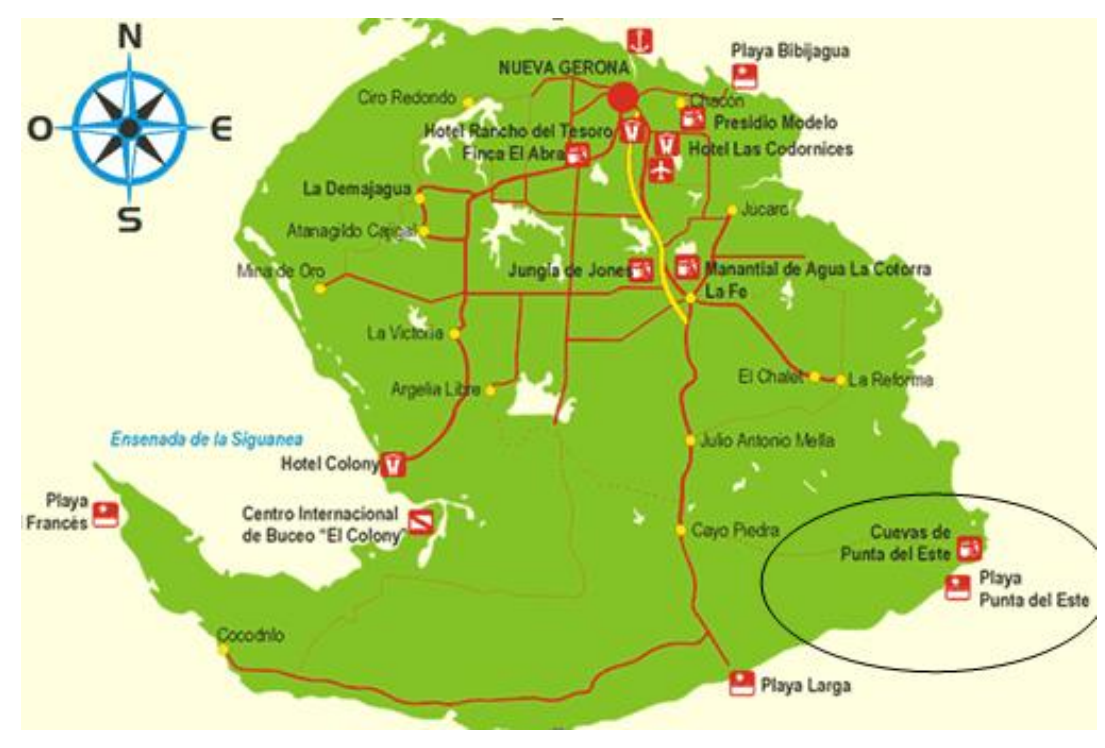

Fuente: Hisae, 2016

En esta zona se encuentra el monumento arqueológico de la Cueva \# 1 de Punta del Este, conocida mundialmente como la "Capilla Sixtina del arte rupestre antillano". Siendo la cueva con mayor cantidad de pictogramas aborígenes del mundo (212 pictogramas), elaborados hace más de 1.000 años por las primitivas tribus Siboneyes que habitaban la zona. Aunque pertenecían al grupo de los Tainos, eran diferentes a las del resto de Cuba y tenía su propia lengua (el "arawak") (Lalueza-Fox et al., 2003). 
Figura 3. Pictograma aborigen de la Cueva 1 en Punta del Este

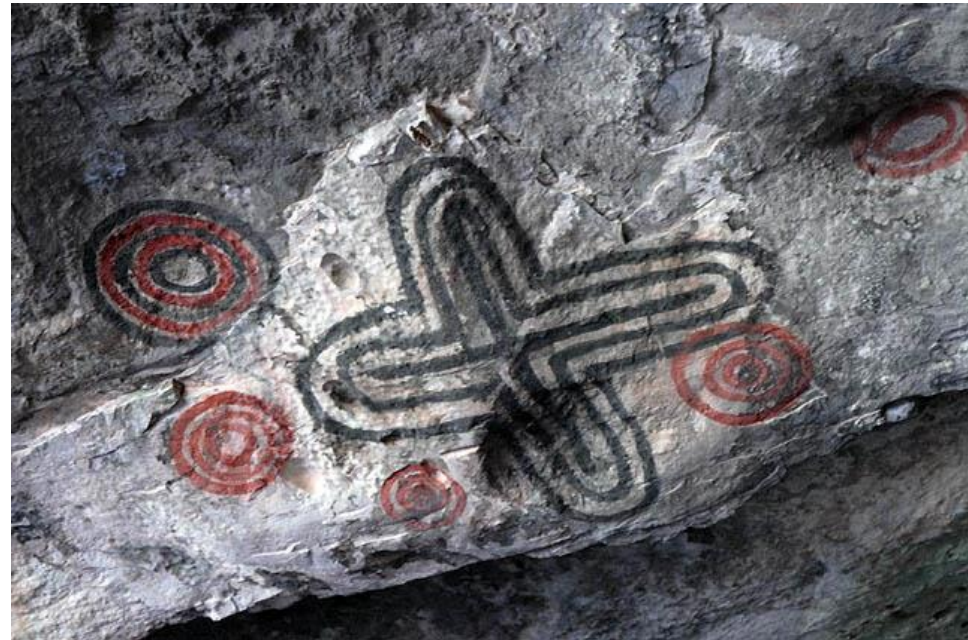

Fuente: Hisae, 2016

La Isla de la Juventud tiene grandes posibilidades para el fomento de la industria turística tradicional, con un mar circundante con más de $35 \mathrm{~km}$ de extensas playas en su costa sur y la de los cayos situados al este.

La riqueza de sus fondos marinos, a lo largo del límite sur de la plataforma insular, es también un recurso de turismo activo inestimable. En conjunto puede llegar a soportar una carga turística numerosa sin llegar a peligrar el entorno, siempre que se desarrolle dentro de las debidas medidas protectoras y con los necesarios profesionales cualificados para controlar y respetar el contexto.

Existen grandes posibilidades de desarrollar en la zona actividades de turismo activo de aventuras en la naturaleza, por la gran cantidad de recursos naturales disponibles, aunque actualmente se desarrollan sin ningún tipo de control. Entre los atractivos más destacados para la práctica de actividades ecoturísticas en esta zona, encontramos las siguientes:

- Sabanas de arenas blancas y en particular en la Reserva Ecológica de "Los Indios".

- Llanura con plantas herbáceas, generalmente de tallo alto y arbustos o árboles aislados sobre roca volcánica en Sabana Grande.

- Alturas residuales y llanuras erosivo-denudativas, con vegetación de pinar y bosques latifolios, en particular en las galerías de la Cañada de los pinares, entre las que destacan el Cerro Monte y la Cañada.

- Elevaciones aisladas del terreno, o mogotes, de roca carbonatada metamórfica.

- Tercio inferior de los ríos principales, con vegetación de galería que transita por la vegetación de manglar en los ecosistemas de los estuarios, con sus tradicionales leyendas de corsarios y piratas.

- Ecosistemas de manglar con esteros y lagunas interiores.

- Ecosistemas de manglar en cayos.

- Flora y Fauna de los cayos, en especial Cantiles y Rosario.

- Ciénaga de Lanier.

- Llanura cársica del Sur.

- Otros recursos histórico-culturales y antropológicos. 
Algunos eventos naturales que se desarrollan en estos espacios, son atracciones ecológicas con grandes posibilidades para el ecoturismo especializado, como se observa en la siguiente tabla:

Tabla 1. Eventos naturales de atracción ecológica y periodos para las prácticas de ecoturismo

\begin{tabular}{|c|c|}
\hline Eventos naturales & Períodos de prácticas \\
\hline $\begin{array}{l}\text { Período reproductivo de la Cotorra cubana } \\
\text { (Amazona leucocephala palmarum) y de la } \\
\text { Grulla cubana (Grus canadensis nesiotes). }\end{array}$ & Febrero a julio \\
\hline Período reproductivo de los quelonios. & Noviembre a junio \\
\hline $\begin{array}{l}\text { Cambio estacional de la flora en la vegetación de } \\
\text { las sabanas. }\end{array}$ & Noviembre a febrero \\
\hline La llegada de la avifauna migratoria. & Octubre a marzo \\
\hline
\end{tabular}

Elaboración propia

Establecer su capacidad de carga turística es importante, puesto que mediante su aplicación podrá lograrse una adecuada conservación de la zona. Si no se ponen en marcha esas "medidas de amortiguamiento y niveles de tolerancia de suelo, flora y fauna, a lo que se enfrenta el destino, es a la llegada masiva de personas, saturación, ruido, basura, ruptura de ciclos de vida animal, extinción de flora y fauna, contaminación de ríos y playas" (Castellanos, 2012, p.68).

Al mismo tiempo, "los ecosistemas en esos ambientes suelen ser sumamente vulnerables, de ahí la importancia de la planificación a largo plazo que busque mantener la eficacia económica, la conservación ambiental y la equidad social" (McCoy, 2015, p.782).

Existen diversas formas de poder controlar la capacidad de carga de acogida de una zona turística sin llegar a deteriorar sus recursos, reducir los niveles de satisfacción de los visitantes, o incrementar los impactos negativos sobre la sociedad, la economía y la cultura local (Flores y Parra, 2007).

Figura 4. Recorrido de la caminata ecoturística

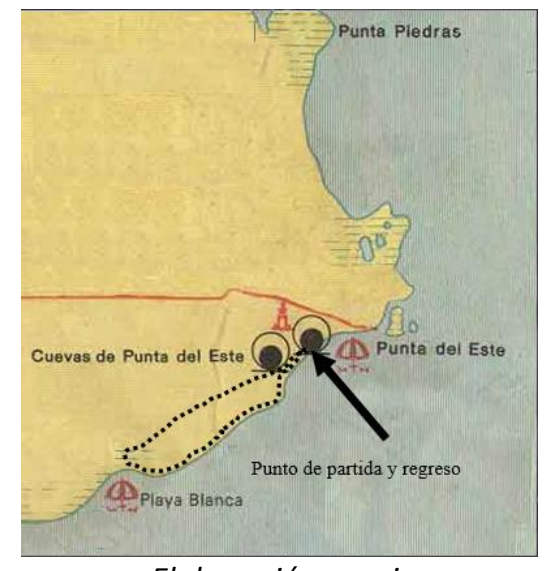

Elaboración propia 


\section{METOdOLOGÍA DE ANÁLISIS DE LA CAPACIDAD DE CARGA EN PUNTA DEL ESTE}

El objetivo del presente estudio es establecer la capacidad de carga de los itinerarios pedestres en la zona de Punta del Este en la Isla de la Juventud (Cuba), teniendo en cuenta la conservación del medio ambiente, el desarrollo socio cultural y la sostenibilidad económica de la zona, como factores necesarios para definir la capacidad de carga para poder "estimar la cantidad óptima de uso del recurso o servicio, manteniendo la condición de equilibrio del sistema natural" (Schlüter y Drummond, 2012, p.998).

El presente análisis es un estudio de caso basado en el método creado por Miguel Cifuentes inicialmente en 1992 y perfeccionado en 1999, en un manual titulado "Capacidad de carga turística de las áreas de uso público del monumento nacional Guayabo, Costa Rica" (Cifuentes, 1999), en el que se buscaba establecer el número máximo de visitas que podía recibir un espacio natural protegido, valorando las condiciones físicas, biológicas y de control que se presentaban en el área en el momento del estudio, a fin de evitar un deterioro de dichos espacios naturales.

El método propuesto por Cifuentes (1999), busca identificar la relación existente entre los niveles de uso de un área natural y los límites cuantitativos que el ecosistema puede soportar. Eso se obtiene por medio de la evaluación de las infraestructuras, equipamientos y personal para la gestión del área natural. Contribuyendo, de esa forma, al equilibrio entre la obtención de ganancias económicas y la protección del patrimonio natural local.

La determinación de la capacidad de carga utilizando el método desarrollado por Cifuentes, aporta información relevante para implantar un plan de control, para conservar el medio natural y aumentar el grado de satisfacción de los visitantes. Además, establece las bases para el desarrollo socioeconómico local por medio del incremento de la actividad ecoturística (Schlüter y Drummond, 2012, p.998).

La presente investigación se realizó mediante la recogida de datos en el lugar de estudio, cumpliendo con todas las normas de información y autorización establecidas a nivel de comité ético para este tipo de estudios con personas. Se aplicó un cuestionario a una muestra de 67 visitantes durante el año 2012. Estos fueron seleccionados aleatoriamente para que, a pesar de su reducido número, garantizaran la ausencia de sesgo en su proceso de selección. Se garantizaba con ello su representatividad para comprobar el perfil socioeconómico, gustos y preferencias de los visitantes.

El tratamiento estadístico de los datos, se realizó mediante un análisis multivariable con el programa Statistical Package for the Social Sciences (SPSS), versión 15.0 para Windows.

Para su mejor comprensión, se indica que las variables de estudio establecidas en este método de investigación son las mencionadas en la obra antes citada (Cifuentes, 1992) y que se van describiendo en este texto a lo largo de los resultados obtenidos en el estudio. No obstante, se han incrementado algunos ítems abiertos, que sirvieron para la triangulación y ampliación de los resultados obtenidos.

El proceso realizado se ha centrado en la zona de Punta del Este y ha constado de tres niveles de análisis: 
- Cálculo de la Capacidad de Carga Física (CCF)

- Cálculo de la Capacidad de Carga Real (CCR)

- Cálculo de la Capacidad de Carga Efectiva (CCE)

Los tres niveles de capacidad de carga tienen una relación que puede representarse de la siguiente manera: CCF $\geq$ CCR $\geq$ CCE. En cuanto a los elementos a considerar para obtener la información básica que permitiera determinar la capacidad de carga de uso turístico, han sido los siguientes:

- Flujo de visitantes en una dirección: Cada persona ocupa un espacio lineal de un metro.

- Distancia mínima entre grupos: 100 metros.

- Tamaño máximo del grupo: 12 pax.

- Duración de la visita: tres horas.

- Longitud de la caminata: 8.795 metros.

- Hay limitaciones por la accesibilidad y la fauna.

- Horario de la visita: 8: 00 AM a 4: 00 PM; es decir, ocho horas diarias.

De la misma manera, existen una serie de factores que se deben considerar como deducciones de la capacidad de carga, como son:

Cada grupo ocupa en hilera 12 metros (1 M x 12 pax.)

El número de grupos se calcula de la forma siguiente:

NG = longitud de la caminata / tamaño del grupo + distancia entre grupos= $8795 /(12$

$+100)=8.795 / 112=78,5$ (cantidad de grupos), se toma 79 por aproximación.

Para determinar el área disponible para el uso de esos 79 grupos se calcula: $A=$ NG $\times$ TMG. A $=79 \times 12=948$ metros (área disponible para el uso).

Las actividades que se desarrollan durante las caminatas serán de tipo lúdico e interpretativo-educativa. Además, estas caminatas no son auto-guiadas, sino que se realizan con guías locales, puesto que el recorrido no está señalizado por el momento.

\section{RESULTADOS}

\subsection{Descripción general del contexto de Punta del Este:}

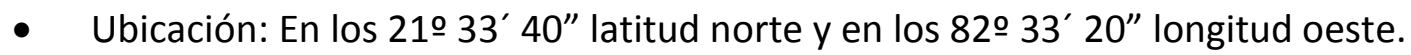

- Área: Punta del Este (Isla de la Juventud, Cuba).

- Acceso: A $60 \mathrm{~km}$ por la carretera de Punta del Este (37 Km por vía asfaltada y $23 \mathrm{Km}$ de tierra).

- Carretera: En mal estado en el último tramo.

- Distancia total del recorrido: $8.795 \mathrm{~m}$.

- Grado de dificultad: Bajo

- Tipo de Caminata: Circular

- Características generales: Ubicada en el extremo Este de la llanura Cárcica del Sur. 
Cuenta con $1.500 \mathrm{~m}$ de playa, con un ancho promedio de $30 \mathrm{~m}$ y la granometría es de medio a fino de color crema claro. Presenta fondo arenoso de pendiente suave.

Como área de sombra cuenta con un uveral, de los mejor conservados y extensos del país, en la que se encuentran cinco formaciones naturales compuestas por los complejos de vegetación de costa rocosa, costa arenosa, bosques de manglares, matorral xeromorfo subcostero y bosque semideciduo micrófilo, especies que se han desarrollado sobre una llanura acumulativa de amplia extensión y cubierta de arena. En esta zona, se encuentra el monumento arqueológico de Punta del Este con los famosos pictogramas en las cuevas de las primitivas tribus Siboneyes (Crispín, 2015).

\subsection{Calculo de la Capacidad de Carga Física (CCF)}

La Capacidad de Carga Física (CCF), es el límite máximo de visitas que se pueden hacer al espacio natural que se analiza en un estudio durante un día. Está determinada por la relación entre factores de visita como el horario y tiempo de visita, el espacio disponible y la necesidad de espacio por visitante. Para su cálculo se utilizó la siguiente fórmula:

$\mathrm{CCF}=(\mathrm{S} / \mathrm{sp}) \times \mathrm{NV}$

En donde:

$\mathrm{S}=$ superficie disponible, en metros lineales $=8.795$ metros.

$\mathrm{sp}=$ superficie usada por persona $=$ un $\mathrm{m}$ de sendero.

NV = número de veces que el sitio puede ser visitado por una misma persona en un día.

En las caminatas equivale a:

$\mathrm{NV}=\mathrm{Hv} / \mathrm{tv}$

$\mathrm{Hv}=$ Horario de visita

Tv = Tiempo necesario para visitar cada sendero.

Con ello se calcula el número de veces que el sitio puede ser visitado por una misma persona en un día (NV), obteniéndose los siguientes resultados:

$\mathrm{NV}=8$ horas $/ 3$ horas. $=2,66$ visitas $/$ días.

Con estos datos se pudo calcular que la Capacidad de Carga Física en esta actividad de turismo activo será en esta zona de:

CCF $=8.795 \mathrm{~m} \times 2,66$ visitas $/$ día $=23.394,7$ visitas/día

\subsection{Cálculo de Capacidad de Carga Real (CCR)}

En cuanto a la Capacidad de Carga Real (CCR), se puede definir como la Capacidad de Carga Física, sometida a una serie de factores de corrección particulares para cada zona, que limitan la visita (Cifuentes, 1999). Los factores de corrección considerados en este estudio fueron: Factor Social (FCsoc), Erodabilidad (FCero), Accesibilidad (FCacc), Precipitación (FCpre), Brillo solar (FCsol) y Cierres temporales (FCctem). Estos factores correctores se aplicaron en función de la fórmula siguiente:

$\mathrm{FCx}=\mathrm{Mlx} / \mathrm{Mtx}$

En donde:

$\mathrm{FCX}=$ Factor de corrección por la variable " $\mathrm{x}$ "

$M \mid x=$ Magnitud limitante de la variable " $x$ "

$M t x=$ Magnitud total de la variable " $x$ " (o metros lineales del espacio) 
a) Factor Social (FCSOc): Consideraron aspectos referentes a la calidad de las visitas, planteándose la necesidad de controlar su realización en grupos. Para un mejor cálculo del flujo de visitantes y a la vez, para asegurar la satisfacción de estos, se propuso que las visitas fueran controladas bajo los siguientes supuestos:

- Grupos de un máximo de 12 personas (pax): Cifuentes (1999) planteó un rango entre 10 y 15 personas por grupos, por lo que se consideró más adecuado en este estudio el tomar un número medio dentro de ese rango y se establecieron 12 personas por cada grupo.

- La distancia entre grupos debe ser de al menos $100 \mathrm{~m}$, para evitar interferencias entre grupos. Cifuentes (1999) planteó una distancia mínima entre grupos de 50 metros, pero en este estudio se asumió que esa distancia debía estar en función del tipo de actividad (lúdicas, interpretativo-educativas, o de aventuras) y el contexto en el que se desarrollase. Por lo que se estableció como más adecuada para estas caminatas educativas una distancia de 100 metros. Al tratarse de una actividad de desarrollo rápido, si no se limita la posibilidad de encuentros entre los visitantes de distintos grupos, puede provocar excesiva concentración de personas en una misma zona.

- Puesto que la distancia entre grupos se consideró como conveniente que fuera de $100 \mathrm{~m}$ y cada persona ocupa $1 \mathrm{~m}$ de sendero, cada grupo requerirá $112 \mathrm{~m}$ de espacio para el desarrollo de la actividad. Por lo que el número de grupos (NG) que pueden estar simultáneamente en este espacio natural desarrollando este tipo de actividad durante un día, será de 79 grupos.

Para calcular el Factor de Corrección Social (FCsoc), es necesario en primer lugar, identificar cuántas personas $(\mathrm{P})$ pueden estar simultáneamente dentro de cada sendero. Para lo que se calculó el número de grupos en función del número de personas por grupo, obteniéndose el siguiente resultado:

$\mathrm{P}=\mathrm{NG} \times$ número de personas por grupo.

$P=79$ grupos $\times 12$ personas $/$ grupo $=948$ personas

Posteriormente, se identificó la magnitud limitante ( $\mathrm{ml}$ ), que en este caso era la porción del sendero que no puede ser ocupada para mantener una distancia mínima entre grupos. Por esto, dado que cada persona ocupa $1 \mathrm{~m}$ del sendero, la magnitud limitante es:

$$
\begin{aligned}
& \mathrm{ml}=\mathrm{mt}-\mathrm{P}=8.795 \mathrm{~m}-948 \mathrm{~m}=7.847 \mathrm{~m} \\
& \text { Con lo que el } \mathrm{FCsoc}=1-(7.847 \mathrm{~m} / 8.795 \mathrm{~m})=1-0.892211483=0.1077
\end{aligned}
$$

b) Erodabilidad (FCero): Dado que este sendero está en su mayor parte cubierto con material relativamente bien consolidado y con pendientes moderadas, se consideraron como limitantes sólo aquellos sectores en donde existían posibilidades o evidencias de erosión, los cuales están identificados como tramos de arena de la playa. Para ello, se calculó el factor de corrección por "erodabilidad" de la siguiente manera:

FCero $=1-(\mathrm{mpe} / \mathrm{mt})$

En donde:

Mpe $=$ metros de sendero con posibles problemas de "erodabilidad" $=1.600 \mathrm{~m}$ $\mathrm{mt}=$ metros totales de sendero $=8.795 \mathrm{~m}$ 
Con lo que el Factor Cero o "erodabilidad" es igual a:

FCero $=1-(1.600 \mathrm{~m} / 8.795 \mathrm{~m})=1-0.1819215463=0.818078$

c) Accesibilidad (FCacc): Mide el grado de dificultad que podrían tener los visitantes para desplazarse por el sendero debido a las pendientes. El análisis de las pendientes de los dos senderos se suele hacer en porcentajes, puesto que es la medida que actualmente se utiliza para realizar esta actividad (Delgado Campuzano et al., 2015). En función de este nivel de dificultad, se establecieron las siguientes categorías:

Tabla 2. Dificultad de las pendientes

\begin{tabular}{ccc}
\hline Pendiente & Grado erodabilidad & Dificultad \\
\hline$\leq 10 \%$ & Bajo & Ningún grado de dificultad \\
\hline $10 \%-20 \%$ & Medio & Dificultad media \\
\hline$>20 \%$ & Alto & Dificultad alta \\
\hline \multicolumn{3}{c}{ Elaboración propia }
\end{tabular}

Los tramos que poseían un grado de dificultad medio o alto, eran los únicos considerados significativos al momento de establecer restricciones de uso. Para ello, se incorporó un factor de ponderación de 1 para el grado medio de dificultad y de 0,5 para el grado bajo de dificultad, puesto que en este tipo de actividad no se presentan niveles altos de dificultad. Con estos datos, el Factor de Accesibilidad de esta actividad se consideró como:

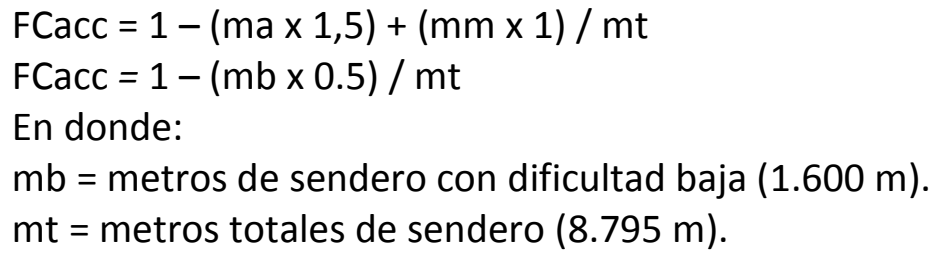

En base a estos datos, el Factor de Accesibilidad de esta actividad en este contexto es el siguiente:

FCacc $=1-(\mathrm{mb} \times 0.5) / \mathrm{mt}=1-(1.600 \mathrm{~m} \times 0.5) / 8.795 \mathrm{~m}=1-0.090960773=$ 0.9090

d) Precipitación (FCpre): Es un factor que impide la visita con normalidad, cuando la actividad se desarrolla bajo la lluvia. Se consideraron los meses de mayo a octubre como los de mayores precipitaciones y en los que la lluvia se presenta con mayor frecuencia en las horas de la tarde y primeras horas de la noche. Aunque no por ello deben de anularse este tipo de actividades de turismo activo de aventuras, puesto que en esas condiciones se pueden encontrar otros tipos de atractivos. A pesar de ello, se determinó que las horas de lluvia limitantes por día en este período, eran de 6 horas (de 2:00 pm a 8:00 pm), lo que representaban 1.098 horas en 6 meses. Con este dato, se calculó el factor de precipitación de la siguiente manera:

FCpre $=1-(\mathrm{hl} / \mathrm{ht})$

En donde:

$h l=$ Horas de lluvia limitantes por año (183 días x 6 hrs $/$ día $=1.098$ hrs)

$h t=$ Horas al año que el espacio está abierto (313 días $\times 8 \mathrm{hrs} /$ día $=2.540 \mathrm{hrs}$ ).

Cifuentes (1999) planteaba que todos los días del año estuviera abierto el espacio 
natural (365 días), pero también estableció que una de las variables que debían tenerse en cuenta era la relacionada con el cierre temporal del área para su recuperación y mantenimiento. Por lo que se calculó que 52 días al año no estaría abierta el área. Se tomó como valor para este cálculo los 313 días, que es la resultante de la resta de 365 días del año, menos los 52 días por cierre operacional. Con ello, se calculó un Factor operacional y de precipitaciones (FCpre) equivalente a:

$$
\text { FCpre }=1-(1.098 / 2.540)=1-0.432283464=0.5677
$$

e) Brillo solar (FCsol): Igualmente que por causa de las lluvias, en algunas horas del día, cuando el brillo del sol es muy fuerte, entre las 10:00 h y las 15:00 h, las visitas a sitios sin cobertura, resultan difíciles o incómodas para los visitantes. Por ello, se tuvo en cuenta esa situación limitante, estableciéndolo como factor reductor. En ese sentido, durante los seis meses con escasa Iluvia, se tomaron en cuenta las cinco horas limitantes (182 días/año x $5 \mathrm{hrs} /$ día = $910 \mathrm{hrs} /$ año) y durante los 6 meses de lluvias sólo se tomaron en cuenta las horas limitantes por la mañana (183 días/año $\times 2$ hrs/día = 366 hrs/año). Estos cálculos sólo se aplicaron a los tramos que transcurrían a pleno sol, sin cobertura de ningún tipo de arbolado. La fórmula aplicada fue la siguiente:

$\mathrm{FCSol}=1-[(\mathrm{hsl} / \mathrm{ht}) \times(\mathrm{ms} / \mathrm{mt})]$

En donde:

$h s l=$ horas de sol limitantes / año (910 h +366 hrs $=1.276$ hrs $)$

$h t=$ horas al año que el área está abierta (2.920 hrs)

$m s=$ metros de caminata sin cobertura $(2.500 \mathrm{~m})$

$m t=$ metros totales de la caminata Punta del Este $(8.795 \mathrm{~m})$

Con esta fórmula se determinó el FCsol con el siguiente resultado :

$\mathrm{FCsol}=1-(1.276 \mathrm{hrs} / 2.920 \mathrm{hrs}) \times(2.500 \mathrm{~m} / 8.795)=1-0.43986301 \mathrm{x}$ 0.284252416

$\mathrm{FCsol}=1-0.125032123=0.8749$.

f) Cierres temporales (FCtem): Por razones de mantenimiento, este espacio natural también tiene previsto no recibir visitantes todos los lunes de la semana, lo que representa una limitación a las visitas en 1 de los 7 días de la semana. Calculándose este factor mediante la siguiente fórmula:

FCtem $=1-(\mathrm{hc} / \mathrm{ht})$

En donde:

$h c=$ Horas al año que el sitio está cerrado $=8 \mathrm{hrs} /$ día $\times 1$ día $/$ semana $\times 52$ semanas/año $=416$ hrs/año.

$h t=$ Horas totales abierto al año $(2.920 \mathrm{hrs})$.

En base a estos datos, el Factor de Cierre Temporal (FCtem) en esta zona para este tipo de actividad es de:

$$
\text { FCtem }=1-(416 \mathrm{hrs} / \text { año } / 2.9220 \mathrm{hrs} / \text { año })=0.8575
$$

Teniendo en cuenta los factores correctores mencionados, se pudo calcular finalmente la Capacidad de Carga Real (CCR), para esta zona en particular y para esta 
actividad específica de las caminatas ecoturísticas, mediante la siguiente fórmula:

$$
\text { CCR }=\text { CCF } \times(\text { FCsoc } \times \text { FCero } \times \text { FCacc } \times \text { FCpre } \times \text { FCsol } \times \text { FCtem })
$$

Obteniéndose como resultado el siguiente:

$$
\begin{aligned}
& C C R=23.394,7 \text { visitas } / \text { día }(0.1077 \times 0.8180 \times 0.9090 \times 0.5677 \times 0.8749 \times 0.8575) \\
& C C R=23.394,7 \times 0.034107062=797,9244833 \text { visitantes por día }
\end{aligned}
$$

\subsection{Cálculo de Capacidad de Carga Efectiva (CCE)}

La Capacidad de Carga Efectiva (CCE), representa el número máximo de visitas que se puede permitir en un espacio natural específico, de forma sostenible y sin producir ningún tipo de deterioro o saturación medioambiental en la zona; o lo que es lo mismo, la Capacidad de Carga Real corregida por la limitación de factores limitantes por la intervención en la zona, como infraestructura, guías, etc. (Cifuentes, 1999).

En primer lugar se calculó la Capacidad de Manejo (CM) para poder calcular la Capacidad de Carga Efectiva (CCE). La capacidad de manejo óptima es definida como el mejor estado o condiciones que la administración de un área protegida debe tener para desarrollar sus actividades y alcanzar sus objetivos. En la medición de la capacidad de manejo (CM), intervienen variables como respaldo jurídico, políticas, equipamiento, dotación de personal, financiación, infraestructura e instalaciones disponibles (Cifuentes, 1999).

En este caso, para realizar una aproximación de la capacidad de manejo de este espacio natural, al tratarse de una propuesta de ofertas recreativas ecoturísticas, se consideraron las siguientes variables: personales, infraestructuras y equipamientos. A tales efectos, en la presente investigación, tras la revisión bibliográfica realizada sobre el tema y las consultas realizadas a numerosos expertos, así como por las propias experiencias profesionales, se determinó otorgar un $15 \%$ de ponderación a este valor. Se ha de aclarar, que en esta zona no existen infraestructuras de atención a los visitantes y que el área de uso para las caminatas no está señalizada. En base a ello, la Capacidad de Carga Efectiva (CCE) se calculó mediante la siguiente fórmula:

$$
\begin{aligned}
& \text { CCE = CCR } \times C M \\
& \text { En donde: } \\
& \text { CCR = Capacidad de Carga Real = } 797.9244833 \\
& \text { CM = Capacidad de Manejo }(15 \%) \\
& \text { Obteniéndose como resultado el siguiente: } \\
& \text { CCE }=797.9244833 \times 15 \%=119.6886724 \text { visitas/día. }
\end{aligned}
$$

\section{DISCUSIÓN DE LOS RESULTADOS}

Mediante el presente estudio de la zona, los datos sobre el impacto que puede soportar este espacio natural sin producirse un deterioro del mismo por causa de las caminatas ecoturísticas, están en función de los resultados que analizamos a continuación. 
Tabla 3. Resultados de cada Capacidad de Carga

\begin{tabular}{cc}
\hline & $\begin{array}{c}\text { Contexto analizado: } \\
\text { Punta del Este }\end{array}$ \\
\hline Capacidad de carga Física (CCF) & 23394,7 \\
\hline Factores de correlación: & \\
\hline FCsoc & 0,1077 \\
\hline FCero & 0,8180 \\
\hline FCacc & 0,9090 \\
\hline FCpre & 0,5677 \\
\hline FCsol & 0,8749 \\
\hline FCtem & 0,8575 \\
\hline Capacidad de Carga Real (CCR) & 797,92 \\
\hline Capacidad de manejo (CM) & $15 \%$ \\
\hline
\end{tabular}

Elaboración propia

Se puede establecer que el número de personas que pueden participar en las actividades de caminatas ecoturísticas desarrolladas en la zona de Punta del Este, sin que se produzca ningún deterioro medioambiental en la zona, es del siguiente:

- Visitantes diarios= 119.68 visitas/días / (2.66 visitas/visitantes/días) = 44.99 visitantes por día que puede soportar el espacio de estudio.

- Visitantes anuales= 44.99 visitantes $/$ día $\times 267$ días $=12.012,33$ visitantes por año.

Mediante estos datos se pudo determinar que probablemente se esté superando en algunas épocas del año la cantidad de visitas recomendadas para superar su capacidad de carga, aunque haciendo el cómputo general de todo el año, esa capacidad no pone en peligro la zona. Aunque habría que comprobar si esa concentración de visitantes en determinadas fechas es permisible mantenerla, o los límites deben calcularse en función del impacto de visitas anuales, puesto que una masificación concentrada en pocos días, puede llegar a producir el mismo daño o más, que su superación a lo largo de todo el año.

\section{CONCLUSIONES}

Este estudio de capacidad de carga, se realizó empleándose como base la metodología establecida por Miguel Cifuentes (1999), aunque se incorporaron algunos elementos correctores de adaptación al contexto para un análisis más amplio, lo que permitió establecer una serie de mejoras y actualizaciones al mencionado método, incorporando algunos ítems abiertos que permitieron completar la información obtenida.

La investigación realizada es un estudio de caso, pero actualmente se trabaja para poder llevar a cabo este mismo estudio en la mayoría de los espacios naturales del país. Se busca poder establecer las diferencias comparativas entre los diferentes espacios naturales, así como entre los diferentes tipos de actividades de ecoturismo y turismo activo que se desarrollan en esos espacios, para poder establecer un Plan nacional de análisis de las capacidades de carga de los espacios naturales. Esto permitiría conocer las limitaciones necesarias para evitar el deterioro de estas zonas por el impacto turístico. Con lo que se 
podría dar un uso más racional y sostenible a los ecosistemas, salvaguardando con ello la riqueza natural de la Isla de la Juventud y de Cuba en general para futuras generaciones.

Los resultados de esta investigación coinciden con los realizados por diversos autores, en el sentido de que la Capacidad de carga en zonas turísticas, "no representa un número mágico que garantice por sí sola la preservación y protección de un área, ni mucho menos resuelve los impactos negativos que la actividad turística pueda llegar a generar dentro de los senderos que se analicen, representa un instrumento único de apoyo para la gestión y conservación de cualquier zona, sea o no un área natural protegida" (Ibáñez Pérez, 2016, p.68).

Como consecuencia de todos los datos obtenidos se llegó a la conclusión final de que, tras el estudio realizado, se ha de recomendar regular la cantidad de visitas para la realización de caminatas ecoturísticas a la zona de Punta del Este en Isla de la Juventud (Cuba), en la siguiente medida:

- Capacidad de carga Física (CCF): En las caminatas ecoturísticas que se realicen en el espacio natural de Punta del Este, el número máximo de visitantes que puede llegar a soportar esta zona es de 23.39 visitantes por día.

- Capacidad de carga real (CCR): Igualmente para esta actividad y en el mismo espacio, teniendo en cuenta la serie de factores que limitan las visitas (como clima, suelo, fauna, etc.), la Capacidad de Carga Real es de 798 visitantes por día.

- Por lo tanto, la Capacidad de carga efectiva (CCF), es decir, la cantidad máxima de visitas que puede soportar la zona para realizar esta actividad sin que se produzca un deterioro del ecosistema, es de 120 visitantes por día.

\section{REFERENCIAS BIBLIOGRÁFICAS}

Castellanos, E. (2012). Planeación del espacio turístico. México: Trillas.

Ceballos-Lascurain, H. (1996). Tourism, Ecotourism and Proted Areas. IV World Congress on National Parks and Protected Areas. Mexico: IUCN-The Word Conservation Union, 301. DOI: 10.2305/IUCN.CH.1996.7.en

Cifuentes, M. (1992). Determinación de capacidad de carga en áreas naturales protegidas. Turrialba (Costa Rica): CATIE.

Cifuentes, M. (1999). Capacidad de carga turística de las áreas de uso público del Monumento Nacional Guayabo, Costa Rica. WWF Centroamérica. Recuperado el 18 de marzo de 2015 de http://www.wwfca.org/wwfpdfs/Guayabo.

Clark, J.R. (1990). Carrying capacity: the limits to tourism. University of Miami. Rosentiel School of marine and atmospheric Sciences. En Congress on Marine Tourism, East/West Conference Center, University of Hawaii, Honolulu, May 23-29.

Crispín, D. (2015). Evolution and analysis of sports tourism and ecotourism in the Isla of the Juventud, Cuba. Journal of Ecosystem \& Ecography, Volume 4 Issue 3, 89. 4th International Conference on Biodiversity, Las Vegas, USA. Recuperado el 18 de marzo de 2015 en http://www.omicsonline.org/ArchiveJEE/biodiversity-2015-posters-andaccepted-abstracts.php. DOI: 10.4172/2157-7625.S1.021.

Delgado Campuzano, D., Herrera Anangonó, R., Villacís Calderón, A., Moreno Vera, A., 
Oviedo

Dunkel, D. R. (1984). Tourism and the Environment: a review the literature and issues. Environmental Society, 37.

Empresa Nacional de Flora y Fauna (1992). Oferta Ecoturística de las Áreas protegidas de Cuba, 13. La Habana.

Flores, P. y Parra, C. (2007). Indicadores de capacidad de carga para el turismo sostenible de la región de Murcia. Encuentros Académicos Internacionales. Recuperado el 21 de marzo de 2015 de http://www.eumed.net/eve/resum/07-07/pfa.htm

Gil, V.N., Gil, V. y Campo, A.M. (2014). Capacidad de carga turística en el sendero del Cerro Ventana. Parque Provincial Ernesto Tornquist, Argentina. Estudios y Perspectivas en Turismo, V. 23, 362-375.

Graefe, A. R., Kuss, F. R. y Vaske, J. J. (1990). Visitor impact management. The planning framework, vol. II, National Parks and Conservation Association, Washington, D. C.

Hisae (2016). Islas del Mundo. Isla de la Juventud. Recuperado el 26 de marzo de 2015) de http://lasislasdelmundo.blogspot.com.es/2016/01/isla-de-la-juventud.html

Holder, J. S. (1988). The pattern and impact of tourism on the environment of the Caribean. En F. Edwards, Tourism in the Caribean. University of Calgary, Canada. Ed. Enviromentally Sound.

Ibáñez Pérez, R. (2016). Capacidad de carga turística como base para el manejo sustentable de actividades ecoturísticas en Unidades de Manejo Ambiental (UMA) de Baja California Sur (BCS). El Periplo Sustentable, 30, 37-76.

Jané, P. (1995). Directorio Turístico de Cuba. Turismo y ecología. Mexico: Limusa, 240-256.

Lalueza-Fox, C., Gilbert, M.T.P., Martínez-Fuentes, A.J., Calafell, F. y Bertranpetit, J. (2003). Mitochondrial DNA from pre-Columbian Ciboneys from Cuba and the prehistoric colonization of the Caribbean. American Journal of Physical Anthropology, V. 121, Issue 2, 97-108. DOI: 10.1002/ajpa.10236

Maldonado, Hurtado, L. y Saborío, O. (1992). Análisis de capacidad de carga para visitación en las áreas silvestres de Costa Rica. Fundación Neotrópica. Centro de Estudios Ambientales y Políticos (CEAP). San José, Costa Rica.

Medina, N. y Santamarina, J. (2004). Turismo de Naturaleza en Cuba. La Habana: Unión, 232.

McCoy, C.E. (2015). Propuesta para recuperar la perspectiva de destino turístico sustentable con base en el análisis de su desempeño y evolución: caso Cancún. Global Conference on Business and Finance Proceedings, V10 (2), 780-789.

Najarro Pujol, L. D. (2011). Cuba por un modelo sostenible como estrategia de desarrollo económico en las cayerías, islotes y parques naturales. RICIT. Revista Turismo, Desarrollo y Buen Vivir, 1.

Organización Mundial del Turismo (2016). Panorama OMT del Turismo Internacional Edición 2015.Datos y Cifras de la Organización Mundial del Turismo. Recuperado el 17 de septiembre de 2016 de http://www.e-unwto.org/doi/book/10.18111/9789284416875

Picornell, C. (1993). Los impactos del Turismo. Papers de Turisme, 11, 65-91.

Sadler, P. (1988). Geometry and stratification of uppermost Cretaceous and Paleogene units on Seymour Island, northern Antarctic Peninsula. Geological Society of America Memoirs, 169, 303-320.

Schlüter, C. y Drummond, J.B. (2012). Evaluación de la capacidad de carga física del Parque Municipal del Itiquira, Formosa (GO), Brasil. Estudios y Perspectivas en Turismo, 21 (4), 996-1012. 
Sierra Maestra (2008). Santiago de Cuba, desarrollo socioeconómico. Dirección Editorial Periódico Sierra Maestra. Recuperado el 17 de junio de 2010 de http://www.sierramaestra.cu/esp/santiago/economia.html.

Soria-Díaz, H.F. y Soria-Solano, B. (2015). Determinación de la capacidad de carga turística en los sitios de visita de la Reserva Nacional Allpahuayo-Mishana, Loreto, Perú. Ciencia amazónica (lquitos), 5 (1), 25-34.

Stankey, G.H., Cole, D.N., Lucas, R.C., Petersen, M.E. \& Frissell, S.S. (1985). The limits of acceptable change. (LAC). System for Wilderness Planning. USDA Forest Service Intermountain Research Station. Ogden. Utah.

Para citar este artículo: Crispin Castellanos, D.; Berovidez Álvarez, V.; Marín Clemente, J.; García Ucha, F.E.; Fernández-

Truan, J.C. (2017). Límites en la capacidad de carga de visitantes de Ecoturismo en Punta del Este, Isla de la Juventud (Cuba). Investigaciones Turísticas, (13), 96-113. http://dx.doi.org/10.461/INTURI2017.13.05 